\title{
THE
}

\section{The Association between Physical Activity and Metabolic Syndrome in Older Adults with Obesity}

\author{
Furong Xu \\ University of Rhode Island, fxu2007@uri.edu \\ Steven A. Cohen \\ University of Rhode Island, steven_cohen@uri.edu \\ Ingrid E. Lofgren \\ University of Rhode Island, ingrid_lofgren@uri.edu \\ Geoffrey Greene \\ University of Rhode Island, ggreene@uri.edu \\ Mifhaethis. Dhelmadmitichal works at: https://digitalcommons.uri.edu/kinesiology_facpubs \\ The University of Rhode Island Faculty have made this article openly available. \\ Please let us know how Open Access to this research benefits you.
}

This is a pre-publication author manuscript of the final, published article.

Terms of Use

This article is made available under the terms and conditions applicable towards Open Access Policy Articles, as set forth in our Terms of Use.

\section{Citation/Publisher Attribution}

F. Xu ; S.A. Cohen ; I.E. Lofgren ; G.W. Greene ; M.J. Delmonico ; M.L. Greaney (2018): The Association between Physical Activity and Metabolic Syndrome in Older Adults with Obesity. The Journal of Frailty and Aging (JFA). http://dx.doi.org/10.14283/jfa.2018.34

Available at: http://dx.doi.org/10.14283/jfa.2018.34

This Article is brought to you for free and open access by the Kinesiology at DigitalCommons@URI. It has been accepted for inclusion in Kinesiology Faculty Publications by an authorized administrator of DigitalCommons@URI. For more information, please contact digitalcommons-group@uri.edu. 


\section{Authors}

Furong Xu, Steven A. Cohen, Ingrid E. Lofgren, Geoffrey Greene, Michael J. Delmonico, and Mary L. Greaney

This article is available at DigitalCommons@URI: https://digitalcommons.uri.edu/kinesiology_facpubs/37 
The Association between Physical Activity and Metabolic Syndrome in Older Adults with

\section{Obesity}

F. Xu ${ }^{1}$, S.A. Cohen ${ }^{2}$, I.E. Lofgren ${ }^{3}$, G.W. Greene ${ }^{3}$, M.J. Delmonico ${ }^{1}$, M.L. Greaney ${ }^{2}$

${ }^{1}$ Department of Kinesiology, University of Rhode Island, Independence Square II,

Kingston, Rhode Island, 02881, United States

${ }^{2}$ Health Studies program, University of Rhode Island, Independence Square II,

Kingston, Rhode Island, 02881, United States

${ }^{3}$ Department of Nutrition and Food Sciences, University of Rhode Island, Fogarty Hall,

Kingston, Rhode Island, 02881, United States

Address correspondence:

Furong $\mathrm{Xu}, \mathrm{PhD}$

Department of Kinesiology

25 West Independence Way, Suite P

The University of Rhode Island

Kingston, RI 02881

Email: fxu2007@uri.edu

Running head: PHYSICAL ACTIVITY AND METABOLIC SYNDROME 


\begin{abstract}
Background: Physical activity reduces the likelihood of developing metabolic syndrome (MetS). However, the association between different physical activity levels and MetS remains unclear in older adults with obesity. Methods: This cross-sectional study used four waves of data (2007-
\end{abstract} 2008, 2009-2010, 2011-2012, 2013-2014) from two datasets: The National Health and Nutrition Examination Survey and United Sates Department of Agriculture's Food Patterns Equivalents Database. The sample included adults $60+$ years of age $(n=613)$ with obesity who had physical activity and MetS data. Physical activity was assessed using the Global Physical Activity Questionnaire and categorized into three physical activity levels (low, medium, and high); and medium or high physical activity levels are aligned with or exceed current physical activity recommendations. Participants were classified as having MetS using a commonly agreed upon definition. Multiple logistic regression models examined the association between the three physical activity levels and MetS risk factors and MetS risk. All analyses adjusted for potential confounding variables and accounted for complex sampling. Results: Of 613 respondents, $72.1 \%$ $(\mathrm{n}=431)$ were classified as having MetS, and 44.3\% $(\mathrm{n}=263)$ did not met physical activity recommendations. Participants with high levels of physical activity had a lower risk of MetS $(\mathrm{OR}=0.31,95 \% \mathrm{CI}: 0.13,0.72)$ and more healthful levels of high-density lipoprotein cholesterol $(\mathrm{OR}=0.39,95 \%$ CI: $0.18,0.84)$, blood pressure $(\mathrm{OR}=0.39,95 \% \mathrm{CI}: 0.20,0.77)$, fasting glucose $(\mathrm{OR}=0.34,95 \% \mathrm{CI}: 0.15,0.78)$ than participants categorized as having low physical activity. Conclusions: Physical activity was associated with lower risk of MetS only for participants with the highest level of physical activity, which suggests that physical activity dosage is important to reduce MetS risk in older adults with obesity.

Keywords: Older adults, obesity, metabolic syndrome, physical activity 


\section{Introduction}

Currently $35.4 \%$ of adults aged $60+$ years of have obesity (body mass index $[\mathrm{BMI}] \geq 30$ $\left.\mathrm{kg} / \mathrm{m}^{2}\right)(1)$. Increasing age and obesity both contribute to metabolic syndrome (MetS) in older adults (2-4). MetS, a predictor of cardiovascular mortality (2), is defined by the presence of at least three of the following risk factors: 1) elevated waist circumference, 2) elevated triglycerides, 3) low high-density lipoprotein cholesterol (HDL-C), 4) elevated blood pressure, and 5) elevated fasting glucose (4). The prevalence of MetS and obesity are increasing concurrently $(5,6)$. About half (46.7\%) of adults 60+ years have MetS (5), and it is estimated that nearly $80 \%$ of older adults with obesity have MetS (6). Research is needed to understand how to better address MetS in this population since rates of MetS are greatest among older adults with obesity $(5,6)$ and older adults are the fast-growing segment of the US population (7).

Physical activity (PA) may prevent or reduce the MetS risk factors as well as the risk of MetS among adults (8-10). Older adults may respond to PA differently than younger adults given that aging-related function declines and various health conditions exist in the older adult population (11). Yet, research examining the relationship between PA and MetS among older adults is lacking (12), and the association between PA dose and MetS in older adults with obesity remains unclear. Furthermore, although physical function limitations may be a barrier to PA participation for all individuals with obesity (13) including older adults (14), the impact of physical function has not been considered in previous studies examining the association between PA and MetS in older adults with obesity. Therefore, the purpose of this study was to examine the association between different levels of PA participation and MetS risk factors and MetS in a representative sample of older adults with obesity independent of physical function limitations. 


\section{Methods}

\section{Study Design}

The present study was a cross-sectional analysis of data from the National Health and Nutrition Examination Survey (NHANES) and United Sates Department of Agriculture's Food Patterns Equivalents Database (USDA-FPED) from 2007 to 2014. Demographic and questionnaire data were collected at respondents' homes. Examination and laboratory data were collected in the Mobile Examination Center (MEC) (15). All data used for the present study were de-identified and are publicly available on the Centers for Disease Control and Prevention and USDA-FPED websites $(15,16)$.

\section{Analytic sample}

The sample was limited to respondents $60+$ years of age $(n=7522)$ with obesity $(23.5 \%$, $\mathrm{n}=1733$ ) whose PA and MetS risk factors data were available. Respondents were excluded if they had physical function limitations and were physically inactive as defined by a zero-minute PA time $(13,15)$. Respondents were classified as having physical function limitations if they reported experiencing any of the following: difficulty or challenges in walking, standing, lifting and carrying things up to 10 pounds, stooping, crouching or kneeling, or that they used special equipment for walking (13). In total, 662 respondents met study inclusion criteria, 49 (7.5\%) of whom were excluded due to lack of PA and/or having physical function limitations, yielding a final analytical sample of 613 respondents.

\section{Physical activity}

PA during a typical week was assessed in three PA domains (work, travel, recreational) using the 16-item Global Physical Activity Questionnaire (17, 18). PA time in each domain was expressed in minutes and summed to create minutes of moderate to vigorous PA per week, then 
converted to metabolic equivalent (MET) minutes which takes PA intensity into account (moderate $\mathrm{PA}=4 \mathrm{MET}$, vigorous $\mathrm{PA}=8 \mathrm{MET}$ ) (18). This information was then used to create three PA categories equivalent to the U.S. Department of Health and Human Services PA guidelines: low (<600 MET-minutes/week), medium (600-1200 MET-minutes/week), and high

(> 1200 MET-minutes/week) (19). The medium or high PA levels are aligned with current PA recommendations ( $\geq 600 \mathrm{MET}$-minutes/week or 150+ minutes of moderate to vigorous PA) (19).

\section{Metabolic syndrome (MetS) parameters}

Five measures were used to classify people as having MetS: triglycerides, HDL-C, fasting glucose, waist circumference and blood pressure. Blood samples were collected at the MEC and then analyzed for triglycerides, HDL-C, plasma glucose at the University of Minnesota following standard laboratory procedures (15). Waist circumference and blood pressure measures were taken at the MEC by trained health technicians using standard methods (15). In addition, participants also reported if they had treatment for triglyceride, or HDL-C, or blood pressure, or blood glucose $(15,20)$. Participants were classified as having MetS if they had three or more of the following: 1) waist circumference $\geq 102 \mathrm{~cm}$ in men or $\geq 88 \mathrm{~cm}$ in women, 2) triglyceride $\geq 150 \mathrm{mg} / \mathrm{dL}$ or with reported lipid lowering treatment, 3) HDL-C $<40 \mathrm{mg} / \mathrm{dL}$ in men or $<50 \mathrm{mg} / \mathrm{dL}$ in women or with reported cholesterol treatment, 4) blood pressure, systolic $\geq 130 \mathrm{~mm} \mathrm{Hg}$ or diastolic $\geq 85 \mathrm{~mm} \mathrm{Hg}$ or both or with reported blood pressure treatment, 5) fasting glucose $\geq 100 \mathrm{mg} / \mathrm{dL}$ or with reported blood glucose treatment $(4,20)$.

\section{Covariates}

Overall diet quality was determined using National Cancer Institute's simple Healthy Eating Index-2015 scoring algorithm using information from two 24-hour dietary recalls data from NHANES and USDA-FPED $(15,16,21)$. Participants were stratified into three tertiles 
based on their total dietary quality score (ranging from 0 to 100) with the top tertile (scores >59.0) being classified as a healthier diet and the other two tertiles (scores $0-46.3$ or 46.3-59.0) being considered indicative of a less healthful diet.

Comorbid disease status was measured using a single question that asked respondents' chronic diseases identified by their doctor or other health professional: arthritis, cancer, cardiovascular disease, chronic kidney disease, diabetes, hypertension, pulmonary disease, and stroke $(15,22)$. A summary score was created ranging from $0-8$ and further divided into four categories: $0,1,2$, or $\geq 3$.

Alcohol consumption was classified into three categories based on respondents' reported alcohol consumption per day in the past year: heavy drinkers, average of $>2$ drinks per day for men and $>1$ drink per day for women; moderate drinkers, average of $\leq 2$ drinks per day for men and $\leq 1$ drink per day for women; never drinkers, no drinking reported $(15,23)$.

Demographic information including age, sex, race/ethnicity, smoking status (never, former, current), education (high school degree or less vs. some college or higher). Respondents also reported their family income and family size, which was then used to calculate poverty to income ratio (PIR) $(15,24)$. PIR was divided into two categories based on the poverty guidelines: at or above (PIR $\geq 1$ ) and below federal poverty level (PIR $<1)(24)$.

\section{Statistical analyses}

The MEC exam 2-year weights were used as the sample weight for all analyses (25).

Baseline characteristics are presented as weighted means \pm standard error for continuous variables and as count and weighted percentages for categorical variables. To assess the associations between PA and MetS risk factors and MetS, the PA was classified into three levels to compare medium and high PA levels with low PA level. Multiple logistic regression models 
were conducted using PROC SURVEYLOGISTIC, including STRATA, CLUSTER, and WEIGHT statement, to obtain adjusted odds ratios (ORs) with 95\% confidence intervals, adjusted for age, sex, race/ethnicity, and current smokers (model 1). As education, poverty levels, alcohol intake, level of comorbidities, BMI, and diet quality may be associated with PA levels, a second logistic regression model was constructed that included these variables as covariates (model 2). Additionally, two sensitivity analyses were conducted in which respondents with physical function limitation were excluded or the drug treatment for elevated triglycerides or low HDL-C as the inclusion criteria to define MetS was excluded, and results did not change substantially. All analyses were performed using SAS 9.4 (SAS Institute Inc., Cary, NC, USA), and p-values $<0.05$ were considered statistically significant.

\section{Results}

Approximately three quarters $(72.1 \%, \mathrm{n}=431)$ of participants were classified as having MetS, $53.5 \%$ of respondents were female, $20.5 \%$ were racial/ethnic minorities, $43.1 \%$ had a high school degree or less and $9.5 \%$ whose household income placed them below the federal poverty line. About half of respondents (44.3\%) were at the lowest PA level and did not met PA recommendations. As seen in Table 1, there were no significant differences in demographic characteristics by MetS status. However, compared to individuals without MetS, participants with MetS were more likely to have a low PA level and more in groups that have $2+$ chronic diseases. In addition, individuals classified as having MetS had larger waist circumference, higher triglycerides, blood pressure, and blood glucose, but lower HDL-C and were more likely to report treatment for lipid profiles and blood glucose.

As shown in Table 2, the multiple logistic models adjusted for age, sex, race/ethnicity and current smoking status (model 1) revealed an inverse association between PA level and the 
risk of MetS ( $\mathrm{p}=0.007)$. That is, respondents classified as having a high PA level were $63 \%$ less likely to have MetS than individuals with low levels of PA (OR $=0.37,95 \%$ CI: $0.20,0.68)$. Although not statistically significant, participants with a medium level of PA were $44 \%$ less likely to have MetS than individuals with low PA $(\mathrm{OR}=0.56,95 \%$ CI: $0.23,1.34)$.

Analyses examining the relationships between PA levels and the five MetS risk factors determined that participants with a high level of PA had a lower risk of having hypertriglyceridemia, low HDL-C, and high blood pressure (see Table 2). The same pattern was observed for HDL-C and blood pressure and participants with a high level of PA had lower risk of elevated fasting glucose ( $\mathrm{OR}=0.34,95 \%$ CI: $0.15,0.78)$ after additionally adjusting for education, poverty levels, alcohol intake, level of comorbidities, BMI, and diet quality (model 2).

Subsequent multiple logistic analyses examined the association of PA levels with MetS risk factors in males and females respectively adjusted for all confounders. Males with a high level of PA had a lower risk of MetS than males with a low level of PA $(\mathrm{OR}=0.33,95 \% \mathrm{CI}$ : 0.14, 0.80). However, females with a high level of PA did not have a lower risk of MetS. In addition, males with a high level of PA had a reduced risk of low HDL-C (OR $=0.35,95 \% \mathrm{CI}$ : $0.13,0.94)$ and elevated blood pressure $(\mathrm{OR}=0.35,95 \% \mathrm{CI}$ : $0.14,0.87)$. Female participants with a high level of PA had lower risk of elevated blood glucose ( $\mathrm{OR}=0.12,95 \% \mathrm{CI}: 0.03,0.51)$ but no other statistically significant differences in MetS risk factors observed (Figure 1).

\section{Discussion}

A large percentage of the sample had MetS (72.1\%) and about half of the respondents not met current PA recommendations (44.3\%). Study findings suggest that more than 1,200 METminutes of PA per week is needed to reduce MetS risk in older adults with obesity who do not 
have physical function limitations. This information is important given the increasing obesity prevalence among older adults and calls to increase PA among older adults (1,8-10).

Study findings indicate that just meeting or slightly exceeding PA recommendations did not reduce MetS risk. The risk of MetS did not decline until PA levels exceeded 1,200 METminutes per week, which equivalent to $42+$ minutes of moderate or $21+$ minutes of vigorous PA per day (18). Studies with adults of all ages have determined that greater levels of PA are associated with a lower MetS risk (8-10). Bell et al. (2015) found that among older adults with obesity those with MetS were less likely to be physically active, although the relationship between PA dosage and MetS risk was not examined and function limitation was not considered (12). The present study extends existing research by focusing on older adults with obesity without physical function limitations and indicates the importance of a PA dosage in excess of 1,200 MET-minutes of PA a week to reduce MetS risk in this population.

A notable finding from the present study is that participants with a high level of PA had a lower risk of low HDL-C, high blood pressure, and elevated fasting glucose. This finding is consistent with previous studies that have found increased HDL-C, lower risk of high blood pressure and elevated fasting glucose associated with higher PA levels although these studies did not focus on older adults with obesity or the US population (8-10). Therefore, comparisons should be made with caution due to the differences in the study populations. Nevertheless, the present study controlled for potential confounders that could biasing the study findings (e.g., diet, level of comorbidities) which support of the benefits of a high PA dosage on MetS risk factors particularly to improve lipid profiles and manage blood pressure and blood glucose, although causality cannot be determined due to the cross-sectional study design. 
Additionally, the present study found that males with a high level of PA had a reduced risk of MetS, HDL-C, and blood pressure while a high PA level in females was only associated with reduced blood glucose. Few studies have examined the association of PA dosage with MetS risk factors or with MetS by sex, and available studies are not specific to older adults with obesity (26-29). Nonetheless, study findings are somewhat consistent with prior research that have observed an inverse relationship between PA and risk of MetS in males (26) and in males and females (27). Previous studies also indicate that a higher PA dosage is associated with lower blood glucose only for males (28) and with higher HDL-C was for females (29). Findings from the current study suggest that among older adults with obesity, males and females may respond to PA dosage differently. Future research should explore these differences.

The present study has strengths and limitations. This is one of first studies to our knowledge, to use nationally representative data to examine the association between different PA levels and MetS among older adults with obesity independent of physical function limitations. In addition, the study assessed three domains of PA (work, travel, and recreational) instead of focusing only on leisure time PA $(8,9)$. Nonetheless, the study is subject to several important limitations including its cross-sectional design that does not allow causality to be determined. PA was assessed by self-report, although a valid instrument was used (17). Also, the study was limited by using existing datasets thus not all possible confounders associated with aging could be examined (e.g., cognitive function, affective status). Additionally, $7.5 \%$ of the eligible sample was excluded due to being physically inactive and/or having physical function limitation. Although, no substantive differences were found with the sensitivity analysis due to this exclusion, this limits our generalizability to older adults with obesity and physical function limitations. 
To conclude, the present study indicates that meeting or slightly exceeding PA recommendations may not be sufficient for lowering MetS risk and only participants with a high PA level (>1,200 MET-minutes/week) had a reduced risk of MetS. Thus, PA dosage might be important to address MetS risk in older adults with obesity and improve public health thus people should be encouraged to exceed PA recommendations if capable but PA promotion needs to be sensitive to gender in different contexts.

Acknowledgement: Data used for the current study were collected by the National Center for Health Statistics, Centers for Disease Control and Prevention and combined from two datasets: The National Health and Nutrition Examination Survey and United Sates Department of Agriculture's Food Patterns Equivalents Database. The content in this paper is the responsibility of the authors and does not necessarily represent the official views of Centers for Disease Control and Prevention or United Sates Department of Agriculture.

Conflict of interest: The authors declare that there are no conflicts of interest regarding this paper. 


\section{References}

1. Ogden CL, Carroll MD, Kit BK et al. Prevalence of childhood and adult obesity in the United States, 2011-2012. JAMA 2014;311(8):806-814. doi:10.1001/jama.2014.732

2. Kaur J. A Comprehensive Review on Metabolic Syndrome. Cardiology Research and Practice 2014; 2014:943162. doi:10.1155/2014/943162.

3. Janssen I. Morbidity and Mortality Risk Associated with an Overweight BMI in Older Men and Women. Obesity 2007; 15: 1827-1840. doi:10.1038/oby.2007.217

4. Alberti KG, Eckel RH, Grundy SM et al. Harmonizing the metabolic syndrome: a joint interim statement of the International Diabetes Federation Task Force on Epidemiology and Prevention; National Heart, Lung, and Blood Institute; American Heart Association; World Heart Federation; International Atherosclerosis Society; and International Association for the Study of Obesity. Circulation 2009;120(16):1640-5.

5. Aguilar M, Bhuket T, Torres $\mathrm{S}$ et al. Prevalence of the Metabolic Syndrome in the United States, 2003-2012. JAMA 2015;313(19):1973-1974. doi:10.1001/jama.2015.4260

6. Wildman RP, Muntner P, Reynolds K et al. The obese without cardiometabolic risk factor clustering and the normal weight with cardiometabolic risk factor clustering: prevalence and correlates of 2 phenotypes among the US population (NHANES 1999-2004). Arch Intern Med 2008; 168:1617-24.

7. Knickman JR, Snell EK. The 2030 Problem: Caring for Aging Baby Boomers. Health Services Research 2002;37(4):849-884. doi:10.1034/j.1600-0560.2002.56.x.

8. Lee J, Kim Y, Jeon JY. Association between physical activity and the prevalence of metabolic syndrome: from the Korean National Health and Nutrition Examination Survey, 1999-2012. Springer Plus 2016;5(1):1870. doi:10.1186/s40064-016-3514-5.

9. Rennie KL, McCarthy N, Yazdgerdi S et al. Association of the metabolic syndrome with both vigorous and moderate physical activity. Int J Epidemiol 2003; 32:600-606. doi: 10.1093/ije/dyg179.

10. Yu Z, Ye X, Wang J et al. Associations of physical activity with inflammatory factors, adipocytokines, and metabolic syndrome in middle-aged and older Chinese people. Circulation 2009; 119: 2969-2977.

11. Stratton JR, Levy WC, Caldwell JH et al. Effects of aging on cardiovascular responses to parasympathetic withdrawal. J Am Coll Cardiol 2003; 41: 2077-2083.

12. Bell JA, Hamer M, van Hees VT et al. Healthy obesity and objective physical activity. The American Journal of Clinical Nutrition 2015;102(2):268-275.

13. Peterson MD, Snih SA, Stoddard J et al. Obesity Misclassification and the Metabolic Syndrome in Adults with Functional Mobility Impairments: NHANES 2003-2006. Preventive medicine 2014; 60:71-76.

14. Paterson DH, Warburton DE. Physical activity and functional limitations in older adults: a systematic review related to Canada's Physical Activity Guidelines. The International 
Journal of Behavioral Nutrition and Physical Activity 2010; 7:38. doi:10.1186/1479-5868-738.

15. Centers for Disease Control and Prevention. National Health and Nutrition Examination Survey. http://wwwn.cdc.gov/nchs/nhanes. Accessed 12 July 2017.

16. United States Department of Agriculture. Food Patterns Equivalents Database. https://www.ars.usda.gov/northeast-area/beltsville-md/beltsville-human-nutrition-researchcenter/food-surveys-research-group/docs/fped-databases/. Accessed 12 December 2017.

17. Armstrong T, Bull F. Development of the world health organization global physical activity questionnaire (GPAQ). J Public Health 2006; 14 (2): 66-70.

18. World Health Organization. Global physical activity questionnaire (GPAQ) analysis guide. http://www.who.int/chp/steps/resources/GPAQ_Analysis_Guide.pdf. Accessed 10 August 2017.

19. The U.S. Department of Health and Human Services. Physical activity guidelines for Americans. http://health.gov/paguidelines/guidelines/chapter5.aspx. Accessed 19 December 2017.

20. Moore JX, Chaudhary N, Akinyemiju T. Metabolic Syndrome Prevalence by Race/Ethnicity and Sex in the United States, National Health and Nutrition Examination Survey, 1988-2012. Preventing Chronic Disease 2017; 14: E24. doi:10.5888/pcd14.160287.

21. National Cancer Institute. The Healthy Eating Index - HEI Scoring Algorithm Method. https://epi.grants.cancer.gov/hei/hei-scoring-method.html. Accessed 14 January 2018.

22. Jindai K, Nielson CM, Vorderstrasse BA, Quiñones AR. Multimorbidity and Functional Limitations Among Adults 65 or Older, NHANES 2005-2012. Preventing Chronic Disease. 2016;13: E151. doi:10.5888/pcd13.160174.

23. Jordan HT, Tabaei BP, Angell SY, Chamany S, Kerker B, Nash D. Metabolic Syndrome Among Adults in New York City, 2004 New York City Health and Nutrition Examination Survey. Preventing Chronic Disease. 2012; 9:E04.

24. U.S. Census Bureau, Population Division, Fertility \& Family Statistics Branch. (2004). Current Population Survey: Definitions and explanations. http:// www.census.gov/population/www/cps/cpsdef.html. Accessed 10 August 2017.

25. CDC National Center for Health Statistics. Specifying weighting parameters. 2013. http://www.cdc.gov/nchs/tutorials/nhanes/surveydesign/weighting/intro.htm. Accessed 11 August 2017.

26. Brien SE, Katzmarzyk PT. Physical activity and the metabolic syndrome in Canada. Appl Physiol Nutr Metab 2006; 31:40-47.

27. Zhu S, St-Onge MP, Heshka S et al. Lifestyle behaviors associated with lower risk of having the metabolic syndrome. Metabolism 2004; 53:1503-1511.

28. Kim J, Choi Y. Physical activity, dietary vitamin C, and metabolic syndrome in the Korean adults: the Korea National Health and Nutrition Examination Survey 2008 to 2012. Public Health 2016 Jun; 135:30-7. 
29. Skoumas J, Pitsavos C, Panagiotakos DB, et al. Physical activity, high density lipoprotein cholesterol and other lipids levels, in men and women from the ATTICA study. Lipids in Health and Disease 2003; 2:3. doi:10.1186/1476-511X-2-3. 
Table 1. Characteristics of subjects with and without metabolic syndrome $(\mathrm{n}=613)$.

\begin{tabular}{|c|c|c|c|c|}
\hline & Total & No metabolic & Metabolic & P-value \\
\hline & $\mathrm{n}=613$ & $\mathrm{n}=182(27.9 \%)$ & $\mathrm{n}=431(72.1 \%)$ & \\
\hline Gender, Male, n (\%) & $296(46.5)$ & $113(61.9)$ & $183(40.5)$ & $<0.001 *$ \\
\hline Age & $69.9 \pm 0.3$ & $70.1 \pm 0.7$ & $69.8 \pm 0.4$ & 0.751 \\
\hline \multicolumn{5}{|l|}{ Ethnicity, n (\%) } \\
\hline NH White & $327(79.5)$ & $96(76.5)$ & $231(80.7)$ & 0.319 \\
\hline NH Black & $118(7.4)$ & $36(7.8)$ & $82(7.3)$ & 0.714 \\
\hline Mexican American & $65(3.4)$ & $22(4.5)$ & $43(3.0)$ & 0.051 \\
\hline Other (including Other & $103(9.7)$ & $28(11.2)$ & $75(9.1)$ & 0.537 \\
\hline \multicolumn{5}{|l|}{ Education level, n (\%) } \\
\hline High school or less & $325(43.1)$ & $98(39.1)$ & $227(44.7)$ & 0.349 \\
\hline College or above & $285(56.9)$ & $83(60.9)$ & $202(55.3)$ & 0.349 \\
\hline PIR above poverty line, n (\%) & $472(90.5)$ & $139(92.3)$ & $333(89.8)$ & 0.301 \\
\hline BMI $\left(\mathrm{kg} / \mathrm{m}^{2}\right)$ & $35.3 \pm 0.3$ & $35.2 \pm 0.5$ & $35.3 \pm 0.3$ & 0.782 \\
\hline \multicolumn{5}{|l|}{ BMI classification } \\
\hline BMI of 30 to $<35 \mathrm{~kg} / \mathrm{m}^{2}$ & $373(62.8)$ & $123(69.9)$ & $250(60.0)$ & 0.064 \\
\hline BMI of 35 to $<40 \mathrm{~kg} / \mathrm{m}^{2}$ & $147(22.8)$ & $35(17.3)$ & $112(25.0)$ & 0.062 \\
\hline BMI of $\geq 40 \mathrm{~kg} / \mathrm{m}^{2}$ & $93(14.4)$ & $24(12.8)$ & $69(15.1)$ & 0.557 \\
\hline \multicolumn{5}{|l|}{ Smoking status, n (\%) } \\
\hline Never smokers & $291(53.3)$ & $98(56.7)$ & $193(51.9)$ & 0.466 \\
\hline Former smokers & $149(25.6)$ & $40(22.1)$ & $109(27.1)$ & 0.318 \\
\hline Current smokers & $105(21.0)$ & $28(21.2)$ & $77(21.0)$ & 0.966 \\
\hline \multicolumn{5}{|l|}{ Alcohol intake, n (\%) } \\
\hline Heavy drinkers & $157(42.2)$ & $49(43.4)$ & 108 (41.7) & 0.777 \\
\hline Moderate drinkers & $143(37.6)$ & $46(38.4)$ & $97(37.3)$ & 0.873 \\
\hline Never drinker & $77(20.2)$ & $22(18.2)$ & $55(21.0)$ & 0.62 \\
\hline \multicolumn{5}{|l|}{ Level of comorbidities, n (\%) } \\
\hline 0 comorbidity & $246(41.2)$ & $105(63.5)$ & $141(32.6)$ & $<0.001 *$ \\
\hline 1 comorbidity & $140(21.1)$ & $47(22.5)$ & $93(20.6)$ & 0.67 \\
\hline 2 comorbidities & $99(16.4)$ & $22(10.8)$ & $77(18.5)$ & $0.045^{*}$ \\
\hline$\geq 3$ comorbidities & $128(21.2)$ & $8(3.2)$ & $120(28.2)$ & $<0.001 *$ \\
\hline \multicolumn{5}{|l|}{ PA } \\
\hline Total PA MET-minutes/week & $3234.2 \pm 305.9$ & $4023.7 \pm 517.9$ & $2929.4 \pm 360.1$ & $0.048^{*}$ \\
\hline Met PA recommendation, $\mathrm{n}(\%)$ & $350(55.7)$ & $121(67.1)$ & $229(51.2)$ & $0.006^{*}$ \\
\hline Low PA, n (\%) & $263(44.3)$ & $61(32.9)$ & $202(48.8)$ & $0.006^{*}$ \\
\hline Medium PA, n (\%) & $58(9.8)$ & $21(9.9)$ & $37(9.8)$ & 0.988 \\
\hline High PA, n (\%) & $292(45.8)$ & $100(57.3)$ & $192(41.4)$ & $0.003 *$ \\
\hline \multicolumn{5}{|l|}{ Diet quality (HEI-2015) } \\
\hline Total diet quality score & $53.4 \pm 0.7$ & $52.4 \pm 1.3$ & $53.8 \pm 0.9$ & 0.382 \\
\hline \multicolumn{5}{|l|}{ Tertile classifications, n (\%) } \\
\hline First tertile $(<46.3)$ & $188(31.4)$ & $54(33.0)$ & $134(30.8)$ & 0.681 \\
\hline Second tertile $(46.3-59.0)$ & $193(34.6)$ & $54(36.7)$ & $139(33.8)$ & 0.658 \\
\hline
\end{tabular}




\begin{tabular}{|lcccc}
\hline Third tertile $(>59.0)$ & $186(34.1)$ & $55(30.3)$ & $131(35.4)$ & 0.344 \\
\hline Metabolic syndrome parameters & & & & \\
\hline Waist Circumference $(\mathrm{cm})$ & $113.0 \pm 0.5$ & $108.4 \pm 1.2$ & $114.7 \pm 0.6$ & $<0.001^{*}$ \\
\hline Triglyceride $(\mathrm{mg} / \mathrm{dL})$ & $126.6 \pm 5.3$ & $90.2 \pm 4.9$ & $155.6 \pm 6.8$ & $<0.001^{*}$ \\
\hline Direct HDL-C $(\mathrm{mg} / \mathrm{dL})$ & $50.3 \pm 0.8$ & $58.2 \pm 1.2$ & $46.8 \pm 1.0$ & $<0.001^{*}$ \\
\hline Systolic $(\mathrm{mm} \mathrm{Hg})$ & $126.4 \pm 0.9$ & $116.9 \pm 1.5$ & $130.0 \pm 1.1$ & $<0.001^{*}$ \\
\hline Diastolic $(\mathrm{mm} \mathrm{Hg})$ & $71.3 \pm 0.7$ & $67.1 \pm 1.4$ & $72.9 \pm 0.7$ & $<0.001^{*}$ \\
\hline Fasting Glucose $(\mathrm{mg} / \mathrm{dL})$ & $111.8 \pm 2.5$ & $100.3 \pm 1.7$ & $120.9 \pm 4.0$ & $<0.001^{*}$ \\
\hline Medication taken & & & & \\
\hline For cholesterol & $143(24.9)$ & $0(0.0)$ & $143(35.2)$ & $<0.001^{*}$ \\
\hline For hypertension & $237(40.3)$ & $17(9.2)$ & $220(52.3)$ & $<0.001^{*}$ \\
\hline For blood sugar & $84(15.6)$ & $6(3.3)$ & $78(20.3)$ & $<0.001^{*}$ \\
\hline
\end{tabular}

Note: Data are present as weighted mean \pm standard error for continuous variables and as count and weighted percentages for categorical variables; $\mathrm{PRI}=$ poverty to income ratio, $\mathrm{BMI}=$ body mass index, PA= physical activity, MET = metabolic equivalent, Low PA = 0-600 MET-minutes/week, Medium PA = 600-1,200 MET-minutes/week, High PA = > 1,200 MET-minutes/week, \# HEI-2015 $=$ Healthy Eating Index $-2015, * \mathrm{p}<0.05$. 
Table 2. The association of physical activity with metaboloc syndrome and metabolic syndrome risk factors $(n=613)$.

\begin{tabular}{|c|c|c|c|c|c|c|}
\hline & \multirow{2}{*}{$\begin{array}{c}\text { No Metabolic } \\
\text { syndrome } \\
\mathrm{n}(\%)\end{array}$} & \multirow{2}{*}{$\begin{array}{l}\text { Metabolic } \\
\text { syndrome } \\
\text { n }(\%)\end{array}$} & \multicolumn{2}{|c|}{ Model $1^{\#}$} & \multicolumn{2}{|c|}{ Model $2^{\&}$} \\
\hline & & & $\begin{array}{l}\text { Adjusted OR } \\
(95 \% \mathrm{CI})\end{array}$ & $\begin{array}{l}\mathrm{P}- \\
\text { value }\end{array}$ & $\begin{array}{l}\text { Adjusted OR } \\
(95 \% \mathrm{CI})\end{array}$ & $\begin{array}{c}\text { P- } \\
\text { value }\end{array}$ \\
\hline $\begin{array}{l}\text { Metabolic syndrome } \\
(\mathrm{n}=613)\end{array}$ & $\mathrm{n}=182$ & $\mathrm{n}=431$ & & & & \\
\hline Low PA & $61(32.9)$ & $202(48.8)$ & Reference & $0.007 *$ & Reference & $0.016^{*}$ \\
\hline Medium PA & $21(9.9)$ & $37(9.8)$ & $0.56(0.23,1.34)$ & & $0.63(0.20,2.01)$ & \\
\hline High PA & $100(57.3)$ & $192(41.4)$ & $0.37(0.20,0.68)$ & & $0.31(0.13,0.72)$ & \\
\hline $\begin{array}{l}\text { Elevated waist } \\
\text { circumference }(n=600)\end{array}$ & $\mathrm{n}=65$ & $\mathrm{n}=535$ & & & & \\
\hline Low PA & $23(36.9)$ & $234(45.0)$ & Reference & 0.485 & Reference & 0.558 \\
\hline Medium PA & $7(9.9)$ & $50(9.8)$ & $0.59(0.14,2.40)$ & & $1.09(0.20,6.10)$ & \\
\hline High PA & $35(53.2)$ & $251(45.2)$ & $0.53(0.18,1.52)$ & & $0.50(0.12,2.22)$ & \\
\hline $\begin{array}{l}\text { Elevated triglyceride } \\
(\mathrm{n}=444)\end{array}$ & $\mathrm{n}=213$ & $\mathrm{n}=231$ & & & & \\
\hline Low PA & $75(34.7)$ & $112(51.5)$ & Reference & $0.027 *$ & Reference & 0.735 \\
\hline Medium PA & $27(11.4)$ & $18(10.5)$ & $0.55(0.26,1.17)$ & & $0.63(0.19,2.04)$ & \\
\hline High PA & $111(53.9)$ & $101(38.1)$ & $0.47(0.27,0.81)$ & & $0.79(0.30,2.08)$ & \\
\hline $\begin{array}{l}\text { Reduced HDL-C } \\
(\mathrm{n}=603)\end{array}$ & $\mathrm{n}=234$ & $n=369$ & & & & \\
\hline Low PA & $90(37.8)$ & $170(48.6)$ & Reference & $0.026^{*}$ & Reference & $0.048 *$ \\
\hline Medium PA & $25(9.6)$ & $32(10.1)$ & $0.66(0.28,1.57)$ & & $0.61(0.21,1.74)$ & \\
\hline High PA & $119(52.6)$ & $167(41.3)$ & $0.46(0.26,0.82)$ & & $0.39(0.18,0.84)$ & \\
\hline $\begin{array}{l}\text { Elevated blood pressure } \\
(n=606)\end{array}$ & $\mathrm{n}=194$ & $\mathrm{n}=412$ & & & & \\
\hline Low PA & $64(32.8)$ & $198(50.6)$ & Reference & $0.001 *$ & Reference & $0.028 *$ \\
\hline Medium PA & $21(10.8)$ & $37(9.5)$ & $0.47(0.21,1.03)$ & & $0.58(0.16,2.06)$ & \\
\hline High PA & $109(56.4)$ & $177(40.0)$ & $0.39(0.24,0.64)$ & & $0.39(0.20,0.77)$ & \\
\hline $\begin{array}{l}\text { Elevated fasting glucose } \\
(\mathrm{n}=414)\end{array}$ & $\mathrm{n}=144$ & $\mathrm{n}=270$ & & & & \\
\hline Low PA & $53(35.6)$ & $126(48.5)$ & Reference & 0.065 & Reference & $0.022 *$ \\
\hline Medium PA & $16(9.9)$ & $24(8.6)$ & $0.70(0.28,1.76)$ & & $0.92(0.35,2.42)$ & \\
\hline High PA & $75(54.6)$ & $120(42.8)$ & $0.50(0.28,0.90)$ & & $0.34(0.15,0.78)$ & \\
\hline
\end{tabular}

Note: \# Model 1: Adjusted for age, sex, race/ethnicity, and current smokers; \& Model 2: Additionally adjusted for education levels, poverty levels, alcohol intake, level of comorbidities, BMI, and diet quality; HDL-C= highdensity lipoprotein cholesterol, PA= physical activity, Low PA: 0-600 MET-minutes/week, Medium PA: 6001,200 MET-minutes/week, Higher PA: > 1,200 MET-minutes/week, * $\mathrm{p}<0.05$. 


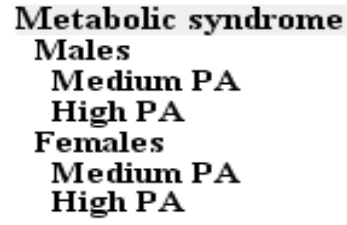

Elevated waist circumference

Males

Medium PA

High PA

Females

Medium PA

High PA

\section{Elevated Triglyceride \\ Males \\ Medium PA \\ High PA \\ Females \\ Medium PA \\ High PA}

\section{Reduced HDL-C \\ Males \\ Medium PA \\ High PA \\ Females \\ Medium PA \\ High PA}

\section{Elevated blood pressure \\ Males \\ Medium PA \\ High PA \\ Females \\ Medium PA \\ High PA}

Elevated fasting glucose

Males

Medium PA

High PA

Females

Medium PA

High PA

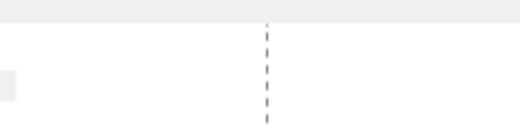

$0.34(0.07,1.55)$

0.33 (0.14, 0.80$)$ *

$0.55(0.07,4.51)$

$0.28(0.08,1.02)$

$1.09(0.19,6.14)$

$0.52(0.14,2.02)$

$1.09(0.19,6.14)$

$0.52(0.14,2.02)$

$0.77(0.17,3.54)$

$0.98(0.39,2.47)$

0.19 (0.04, 0.87)*

$0.84(0.22,3.20)$

$0.39(0.11,1.37)$

0.35 (0.13, 0.94)*

$0.80(0.19,3.39)$

$0.56(0.19,1.70)$

0.33 (0.08, 1.45)

$0.35(0.14,0.87)$ *

0.71 (0.10, 4.99)

$0.37(0.12,1.13)$

0.942

0.04 *

0.504

0.504

0.048 *

0.579

0.049*

0.216

0.946

1.25 (0.10, 15.65)

$0.89(0.23,3.49)$

$0.62(0.10,3.88)$

$0.011^{*}$

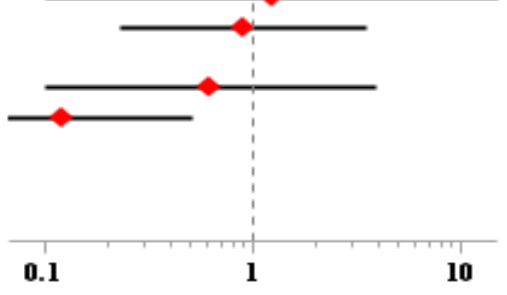

$.12(0.03,0.51)^{*}$

Figure 1. The association of physical activity and metabolic syndrome by males and females.

PA= physical activity, HDL-C= high-density lipoprotein cholesterol, Medium PA: 600-1,200 METminutes/week, High PA: > 1,200 MET-minutes/week, Low PA: 0-600 MET-minutes/week and Low PA is reference category, all analyses adjusted for age, race, education level, household income, current smokers, alcohol intake, level of comorbidities, BMI, and diet quality, $* \mathrm{p}<0.05$. 